\title{
Mean-field analog of the Hong-Ou-Mandel experiment with bright solitons
}

\author{
Zhi-Yuan Sun \\ Department of Physics, Technion - Israel Institute of Technology, Haifa, Israel \\ Panayotis G. Kevrekidis \\ Department of Mathematics and Statistics, University of Massachusetts, Amherst, Massachusetts 01003, USA \\ Peter Krüger \\ Midlands Ultracold Atom Research Centre, School of Physics \& Astronomy, The University of Nottingham, \\ Nottingham NG7 2RD, United Kingdom
}

(Received 29 July 2014; revised manuscript received 4 November 2014; published 5 December 2014)

\begin{abstract}
In the present work, we theoretically propose and numerically illustrate a mean-field analog of the Hong-OuMandel experiment with bright solitons. More specifically, we scatter two solitons off of each other (in our setup, the bright solitons play the role of a classical analog to the quantum photons of the original experiment), while the role of the beam splitter is played by a repulsive Gaussian barrier. In our classical scenario, distinguishability of the particles yields, as expected, a 0.5 split mass on either side. Nevertheless, for very slight deviations from the completely symmetric scenario, a near-perfect transmission can be constructed instead, very similarly to the quantum-mechanical output. We demonstrate this as a generic feature under slight variations of the relative soliton speed, or of the relative amplitude in a wide parametric regime. We also explore how variations of the properties of the "beam splitter" (i.e., the Gaussian barrier) affect this phenomenology.
\end{abstract}

DOI: 10.1103/PhysRevA.90.063612

PACS number(s): 03.75.Lm, 05.45.Yv

\section{INTRODUCTION}

The Hong-Ou-Mandel (HOM) effect is, by now, a wellestablished experiment in quantum mechanics that describes two-particle interference based on a pair of indistinguishable photons [1]. When those two identical single-photon wave packets simultaneously enter a 50:50 beam splitter, one in each input port, both always exit the splitter at the same output port, although each photon has (on its own) a 50:50 possibility to exit either output port. As a result of this effect, we can test the degree of indistinguishability of two incoming photons experimentally. While a direct measurement of both quantum particles (photons) exiting the beam splitter through the same port is normally not possible, the number of coincidence counts of the photons exiting the beam splitter through one exit port each dips to zero (the so-called HOM dip) in the case of perfect indistinguishability. Santori et al. applied the HOM effect to demonstrate the purity of a solid-state single-photon source [2], while Beugnon et al. experimentally considered two atoms independently emitting a single photon to produce the HOM effect [3]. From the point of view of applications, the HOM effect has provided a mechanism for logic gates in linear optical quantum computation [4]. It is important here to stress the fact that in the case where solitonic wave packets replace the photons, in contrast, a direct measurement does become possible and a classical mean-field description, as used in this work, can be employed.

As a generalization of the HOM effect, recent studies have been devoted to the interference of massive particles. Lim and Beige have considered HOM experiments with $N$ bosons or fermions passing simultaneously through a symmetric Bell multiport beam splitter [5]. Longo et al. have examined the joint probability distribution (of finding both photons on the same side) upon varying the properties of the beam splitter [6]. Laloë and Mullin generalized the HOM effect for a large number of particles by investigating quantum properties of a single beam splitter [7]. In fact, Bose-Einstein condensates (BECs) at very low temperatures provide a setup for studying an analog to the HOM effect for massive particles. Recently, Lewis-Swan and Kheruntsyan proposed the realization of the HOM effect for massive particles by using a collision of two BECs and a sequence of laser-induced Bragg pulses as the splitter [8]. (Such an experimental technique has been formerly used to demonstrate the violation of the CauchySchwarz inequality with matter waves [9].) This has been further explored experimentally very recently in a plasmonic setup using surface plasmon polaritons (SPPs) [10]. Here the two photons were converted into SPPs on a metal stripe waveguide and were subsequently made to interact through a semitransparent Bragg mirror. This resulted in a coincidence count dip by $72 \%$, confirming the bosonic nature of the SPPs and the HOM destructive quantum interference effect.

On the other hand, on the more "classical" side of matter waves, bright solitary waves or solitons have been extensively studied in the context of Bose-Einstein condensates [11]. Restricting our consideration (for the present work) to attractive interactions and bright solitary waves, we can note that both one [12] and many [13,14] (as a result of the modulational instability) such waves have been created in ${ }^{7} \mathrm{Li}$. More recently, they have also been produced in ${ }^{85} \mathrm{Rb}$ [15], and furthermore the interactions between them and with barriers have been explored [16-19] both at the mean-field and at the quantum-mechanical $[20,21]$ level. Very recently experimental signatures have also been reported, both for the case of interactions with barriers [22] and for those between bright solitons with different phases [23]. It should be noted here that while bright solitons $[16,24]$ and even their trains [25] and collisions [26] constitute well-established themes in the BEC literature, interesting variants thereof continue to emerge, including bright solitons in spin-orbit $[27,28]$ and 
exciton-polariton BECs [29,30]. Moreover, new experimental techniques for their production (such as $\mathrm{rf}$ evaporation for producing one or a pair of bright solitons) [31] and their use in applications including interferometry [32] are being devised.

It is at the junction of these two exciting research themes that the present study treads. The bright solitary waves possess some quantum-mechanical (or, more accurately, wavelike) features, including a transmission and reflection from a repulsive Gaussian barrier (which we will consider hereafter) as has been analyzed physically in [18], based to a large extent on the authoritative earlier mathematical analysis of [33]. However, they are not genuine quantum particles such as photons superposed in Fock states. A manifestation of the latter feature is the fact that the HOM dip occurs when the photons are perfectly indistinguishable. On the other hand, if two identical bright solitons enter the beam splitter perfectly symmetrically, the result of their collision will be a perfect splitting into an output state, with one soliton emerging from each port.

Nevertheless, what we argue here is that our mean-field treatment shows that even very slight deviations of the bright solitons from perfect symmetry (of the order of a few percent in the relative speed, or in the relative amplitude) yield an output whereby the bright solitons emerge in only one of the two (controllably so, depending on the sign of the asymmetry) ports, i.e., as an analog of the $|2,0\rangle$ or the $|0,2\rangle$ state. Indeed, it is this analogy with the HOM feature of revealing both "particles" (in our case, the bright solitons) on one side which constitutes a remarkable feature that arises over a wide range of values of both the soliton and the barrier parameters and as such can be considered as generic. We argue that this phenomenon cannot purely emerge from the interaction of the bright solitons with the barrier but must stem critically from their pairwise interaction during their "coincidence" at the barrier. These deviations in soliton parameters (that give rise to these asymmetries) are so weak that they can very straightforwardly arise due to the imperfect preparation of the colliding bright solitons. Thus the interference of our (very) weakly asymmetric bright solitons at the barrier is responsible for their emergence on one or the other port in this setting.

Our presentation is structured as follows. In Sec. II, we briefly discuss the theoretical setup. Section III contains our numerical results and a discussion of the variation of our phenomenology over regimes of speed and amplitude variations of the bright solitons and amplitude and width variations of the barrier. Finally, Sec. IV contains a summary of our findings and a number of suggestions for potential future studies.

\section{THEORETICAL SETUP}

We set two bright solitons of BECs to collide at a narrow barrier of Gaussian form, which is viewed as the analog of the beam splitter. We should note here that this kind of setup has been extensively studied recently. The thorough exposition and analysis of [18] explored the outcome of the collision of two identical bright solitons, examining chiefly and even semianalytically, following the work of [33], the role of asymmetries in the phase between the bright solitons (Sec. IV A therein). The issue of asymmetry in bright soliton amplitudes was briefly discussed as well (in Sec. IV B therein) without a special focus, to the best of our understanding, on the phenomenology reported here. In particular, the central question (here) of the outcome of uneven bright soliton velocities was not considered in [18]. On the other hand, the work of [19] reports observations very similar to the focus of the present work. (See, e.g., Fig. 8 of Ref. [19] and in particular, the evolution of the two bright solitons colliding after $t=0.1 \mathrm{~s}$ in the top panel.) Nevertheless, as this work concerned the collision of a single bright soliton with a barrier (where the interaction of two bright solitons with the barrier was only a secondary effect, due to the "return interaction" of the two splinters), this feature was not examined systematically, although glimpses of it can be inferred by the second reflection coefficient plots of Fig. 5 in [19].

To simplify the relevant context, we examine the collision phenomenology in the setting of the normalized quasi-onedimensional Gross-Pitaevskii (GP) equation with attractive interactions:

$$
\begin{aligned}
i \frac{\partial \psi(x, t)}{\partial t}= & {\left[-\frac{1}{2} \frac{\partial^{2}}{\partial x^{2}}+\frac{q}{\sigma \sqrt{2 \pi}} e^{-x^{2} /\left(2 \sigma^{2}\right)}-|\psi(x, t)|^{2}\right] } \\
& \times \psi(x, t),
\end{aligned}
$$

where $\psi(x, t)$ is the dimensionless wave function with normalized temporal and spatial coordinates $t$ and $x$; see, e.g., [18] for a discussion of the relevant units. The dimensionless form of the equation has been systematically derived, e.g., in [11]. (See Chap. 1 therein, while Chap. 2 is specifically dedicated to bright solitons.) While, as discussed in [19], quantitative features may be expected to differ in the three-dimensional (3D) case (and the latter may also differ even qualitatively for near-critical atom numbers in the vicinity of the collapse threshold), qualitative features of the one-dimensional (1D) GPE can be fairly accurate for a wide range of atom numbers (up to $\approx 0.7 N_{c}$, where $N_{c}$ is the critical atom number); see the comparison in Fig. 1 of Ref. [19].

The Gaussian barrier has a normalized width $\sigma$ and strength $q$. Our initial condition in the present work involves two oppositely moving bright solitons of the form

$$
\begin{aligned}
\psi(x, t=0)= & k_{1} \operatorname{sech}\left[k_{1}\left(x+x_{1}\right)\right] e^{i v_{1} x} \\
& +k_{2} \operatorname{sech}\left[k_{2}\left(x-x_{2}\right)\right] e^{-i\left(v_{2} x+\Delta\right)} .
\end{aligned}
$$

For sufficiently large values of $x_{1}$ and $x_{2}\left(x_{1,2}>0\right)$, Eq. (2) approximately represents a pair of two bright solitons located at $-x_{1}$ and $x_{2}$, with amplitudes $k_{1}$ and $k_{2}$, oppositely moving velocities $v_{1}$ and $-v_{2}\left(v_{1,2}>0\right)$, and with a relative phase $\Delta$. Although this ansatz is reminiscent of the one used in [18], contrary to that study here we will generally not utilize the phase difference, setting it to $\Delta=0$, unless indicated otherwise; a relevant brief comment on its role is included in the next section. Instead, a critical distinguishing feature of the present work will be that we will be relying on slight asymmetries of the propagation characteristics of the solitons, such as their speeds or their amplitudes/inverse widths, in order to achieve our mean-field analog of the HOM effect. Note that the limiting case of a $\delta$-shaped barrier $(\sigma \rightarrow 0$ for the Gaussian barrier) has been treated analytically by Holmer et al. [33] for a single bright soliton. 
As our mean-field experiment, with the two bright solitons playing the role of (partially classical) analogs of the photons in the original HOM setting, and the barrier acting as the beam splitter, we arrange for the two matter-wave bright solitons located at $-x_{1}$ and $x_{2}$ to collide exactly at the center of the Gaussian barrier, which requires $x_{1} / v_{1}=x_{2} / v_{2}$. The essential point is to control a slight difference between the velocities $v_{1,2}$. In our setup, we ensure that $\left|\frac{v_{2}-v_{1}}{v_{1}}\right| \leqslant 0.1$. As numerical diagnostics of the "mass" (i.e., atom number fraction) that emerges on each side as a result of this experiment, we compute two normalized integral quantities:

$$
E_{+}=\frac{\int_{0}^{+\infty}|\psi|^{2} d x}{\int_{-\infty}^{+\infty}|\psi|^{2} d x}, \quad E_{-}=\frac{\int_{-\infty}^{0}|\psi|^{2} d x}{\int_{-\infty}^{+\infty}|\psi|^{2} d x}
$$

It is then well known (and easily understood by symmetry) that for $v_{1}=v_{2}$ and other parameters chosen the same for both incoming bright solitons, it will be true by construction that $E_{+}=E_{-}=0.5$. We now turn to the case of unequal velocities and amplitudes in our computations presented below.

\section{NUMERICAL RESULTS AND DISCUSSION}

In the case of unequal velocities, we illustrate typical realizations in Fig. 1. (The numerical simulation is performed using a fourth-order Runge-Kutta algorithm in time, secondorder centered difference in space scheme.) As seen (and in line with observations such as the secondary collision of Fig. 7 in [19]), the small difference $(\approx 2 \%)$ in the bright soliton velocities induces a dramatic deviation from the abovementioned equal velocity result. In other words, envisioning two detectors located on the bright soliton moving paths after collision, we observe that essentially a sole, double-in-mass solitary wave packet will be found at one detector, with nearly no mass collected at the other one. (The partition is nearly $99 \%$ and $1 \%$ in the example shown in Fig. 1.) In the HOM Fock space language, a $|2,0\rangle$ or $|0,2\rangle$ state (cf. Fig. 1) is recovered rather than a coincidence count of $|1,1\rangle$, in some sense similarly to the genuine quantum particle result. Unlike the quantum case, the entangled state $[|2,0\rangle+|0,2\rangle] / \sqrt{2}$ is not what is found here. Instead, the slight asymmetry determines uniquely on which output port of the beam splitter the double-mass bright soliton is found, thus replacing the decoherence process of the quantum case.

Figure 2 examines the role of the difference between $v_{1}$ and $v_{2}$ by fixing $v_{1}=1$, and varying $v_{2}$ in a small range of 0.9-1.1. The simulation results, as illustrated by the quantities $E_{+,-}$, are shown in the figure. We see that a peak value occurs when $v_{2} / v_{1}$ approaches 1 from lower values, which means most of the bright soliton energy is found in one detector. The situation is symmetric as $v_{2} / v_{1}$ approaches 1 from higher values, and the energy (peaking again around 99\%) is detected in the other output port this time. The remarkable feature is that for such a dramatic effect, miniscule deviations of the order of only $1 \%-2 \%$ are sufficient. As observed in Fig. 2, the asymmetry is maintained above $70 \%-30 \%$ nearly throughout our parametric interval of observation. The tiny region near $v_{1}=v_{2}$ is an exception that is explainable by the setup of our model (see above). Experiments are eventually expected to show whether this result prevails empirically. If it does,

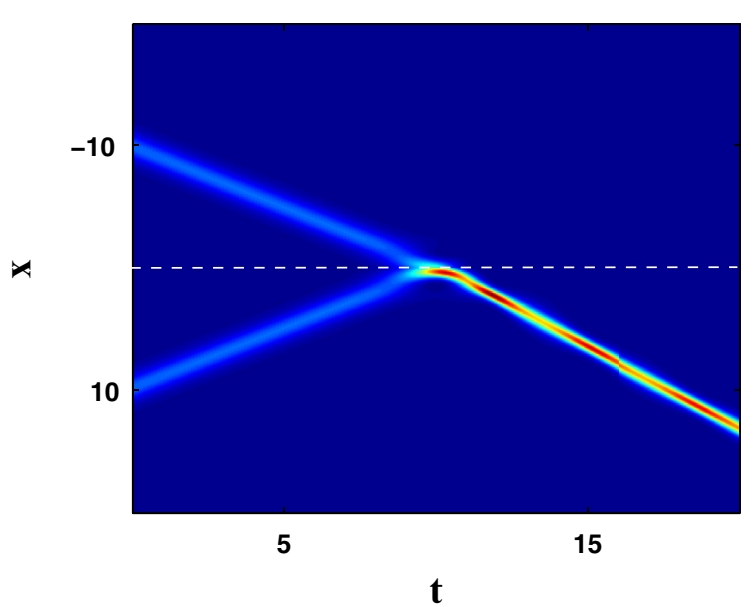

(a)

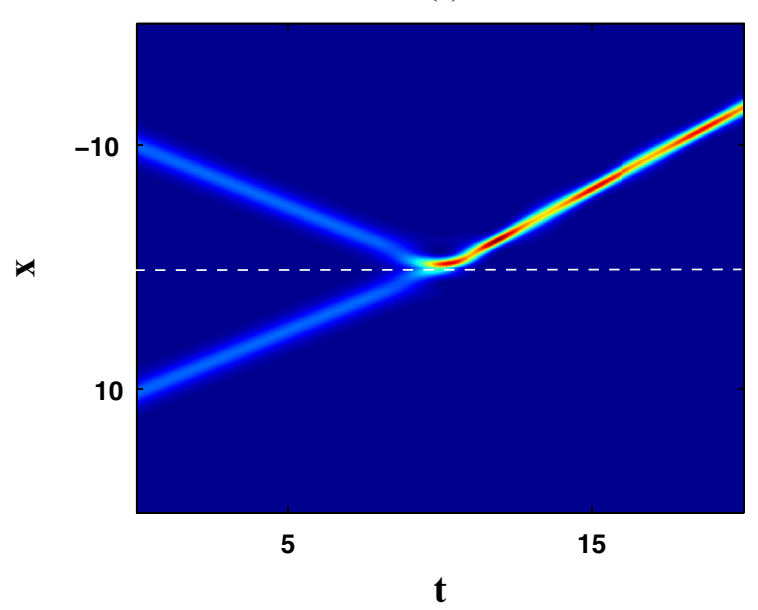

(b)

FIG. 1. (Color online) Numerical simulation of the HOM analogous effect for a two (slightly asymmetric) matter-wave bright soliton collision at the Gaussian potential barrier. The parameters are chosen as $q=1, \sigma=0.1, k_{1}=k_{2}=1$, and $\Delta=0$. (a) $v_{1}=1.00, v_{2}=0.98$, and $x_{1} / v_{1}=10$; (b) $v_{1}=1.00, v_{2}=1.02$, and $x_{1} / v_{1}=10$.

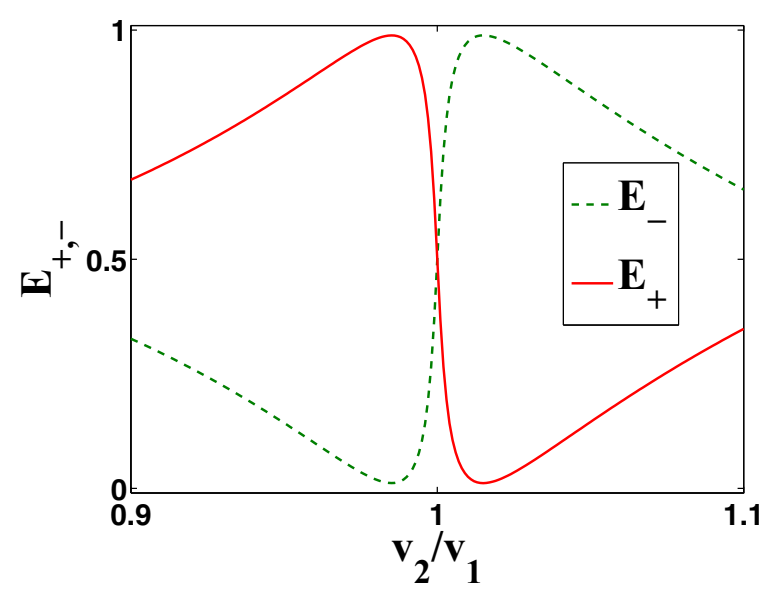

FIG. 2. (Color online) Plots of $E_{+,-}$[see Eq. (3)] as a function of $v_{2} / v_{1}$ varying from 0.9 to $1.1\left(v_{1}=1\right)$. The parameters are chosen as $q=1, \sigma=0.1, k_{1}=k_{2}=1, \Delta=0$, and $x_{1} / v_{1}=x_{2} / v_{2}=20$. 
the narrowness of the feature could open the path to using bright soliton collisions for sensitive measurements of minute external forces modifying the effective travel path length. Aside from this narrow region, it can be observed that a situation most closely reminiscent of the original HOM output is best replicated when the bright solitons have a roughly $50 \%$ transmission and reflection probability from the barrier; this will be further illustrated in what follows.

We should note here that for a single bright soliton, the numerical results of Helm et al. [18] suggest that, in the approximate range $0.5 \leqslant v \leqslant 2$ and $\sigma \leqslant 0.28$, the bright soliton should tunnel through the Gaussian barrier instead of classically passing through. As a result, the authors conclude that the following relation is satisfied within this tunneling regime:

$$
\frac{1}{2} v^{2} \ll \frac{q}{\sigma \sqrt{2 \pi}} .
$$

This translates into $v \ll 2.8$ for $q=1$ and $\sigma=0.1$ to achieve the tunneling regime.

In Fig. 3 we illustrate how the effect of the slight velocity difference explored above (in analogy with HOM) is modified for faster, as well as more slowly moving bright solitons within this tunneling regime. Several features can be distinguished here. First, there is an "optimum" with regard to the mass asymmetry involved in the process. This appears to arise when $v_{1} \approx q$. This, in turn, suggests a nearly 50:50 beam splitter, given the expressions for transmission and reflection from a $\delta$ barrier [cf., e.g., Eqs. (7)-(10) and associated discussion in [18]]. Second, the location of the maximal asymmetry is monotonically approaching $v_{2} / v_{1}=1$ as $v_{1}$ is decreasing. Nevertheless, and while for large $v_{1}$ the phenomenon is more pronounced, with a rapid decrease of asymmetry as $v_{2} / v_{1}$ deviates more significantly from unity, the opposite is true in the slow case. For small $v_{1}$ (slow, bright solitons), although the peak approaches $v_{2} / v_{1}=1$, the curve also flattens and becomes nearly insensitive to the exact value of $v_{2} / v_{1}$ and the corresponding asymmetry is far less pronounced. From the above, we infer that this asymmetry is most evident when $v_{1}$ (and $v_{2}$ ) are near $q$ and the relevant mass peak in that case is very proximal to unity, while it occurs only within a few percent of the $v_{1}=v_{2}$ limit. It should be added here that in line with the discussion of [18], such an asymmetry cannot be justified by a brief (rapid) interaction of the solitary waves with the barrier. Exploring the formulation of [33] in the same way as is done in Sec. IV A of [18], it can be shown that the relative mass (the factors of $\left|P_{-}\right|^{2}$ and $\left|P_{+}\right|^{2}$ ) smoothly deviate from $1 / 2$ by only a few percent and hence cannot justify the asymmetry we observed. Thus it must be that this outcome is due to the interaction of the solitary waves with each other and with the barrier. For vanishing intersoliton interaction, each bright soliton would individually interact with the barrier so that slight asymmetries would only cause slight deviations from a 50:50 splitting, always resulting in close to equal population at the two outputs of the beam splitter in the presented setup. Conversely, for a collision event of two bright solitons in the absence (or far away from) the barrier, the interactions would be far too weak to cause a significant deviation from a 50:50 split (for identical or near-identical bright solitons), i.e., the bright solitons would essentially pass

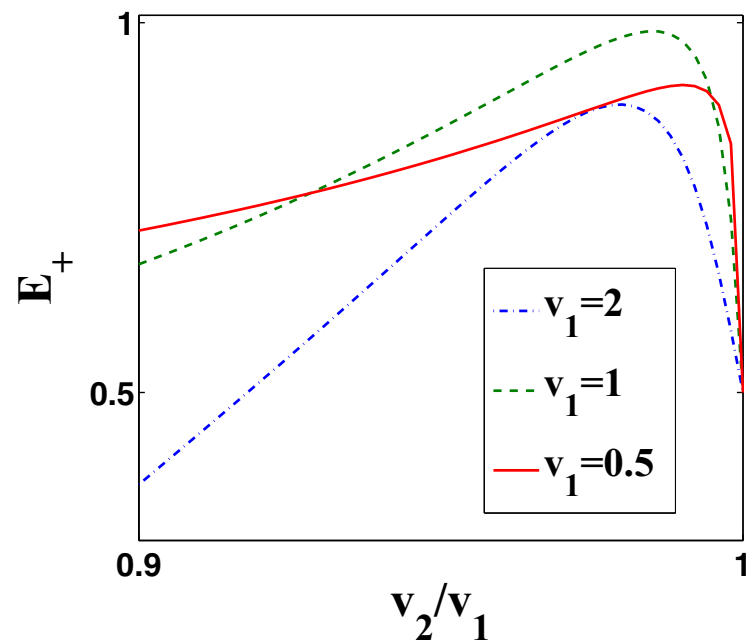

(a)

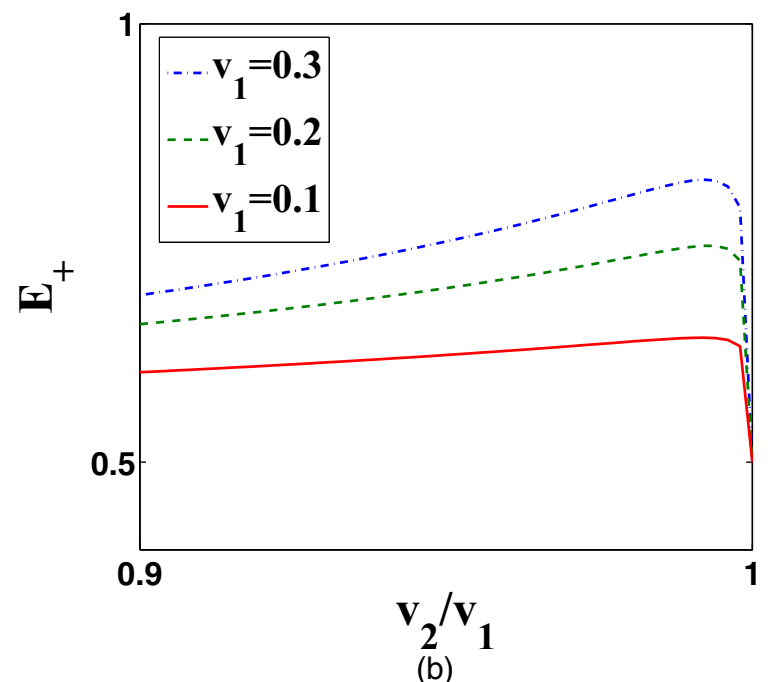

FIG. 3. (Color online) Plots of $E_{+}$[see Eq. (3)] with $v_{2} / v_{1}$ varying from 0.9 to 1.0 for two groups of three values of $v_{1}$ : (a) $v_{1}=0.5,1.0$, and 2.0; (b) $v_{1}=0.1,0.2$, and 0.3. The parameters are chosen as $q=1, \sigma=0.1, k_{1}=k_{2}=1, \Delta=0$, and $x_{1} / v_{1}=$ $x_{2} / v_{2}=20$.

through each other, as they would constitute exact bright solitonic solutions of the integrable 1D homogeneous GP equation.

Although in all other results reported in the manuscript, the relative phase of the bright solitary waves is initially chosen to be $\Delta=0$, given the difficulties in experimentally controlling such a phase, it is, arguably, of relevance to explore the role of $\Delta$ in potentially affecting the above results. This is examined in Fig. 4. In the top panel of the figure, it can be seen that indeed variations in the original phase difference $\Delta$ will shift the speed ratio $v_{2} / v_{1}$ of optimal induced asymmetry (i.e., of maximal $E_{+}$). Nevertheless, as illustrated in the bottom panel, the variation is only linear and its small slope ensures that the phenomenology presented above (and below) will be relevant even in the presence of nontrivial phase differences $\Delta$.

We now consider different variants of the relevant phenomenology. If, for instance, the two bright solitons collide a bit further away from the center of the Gaussian barrier, the 


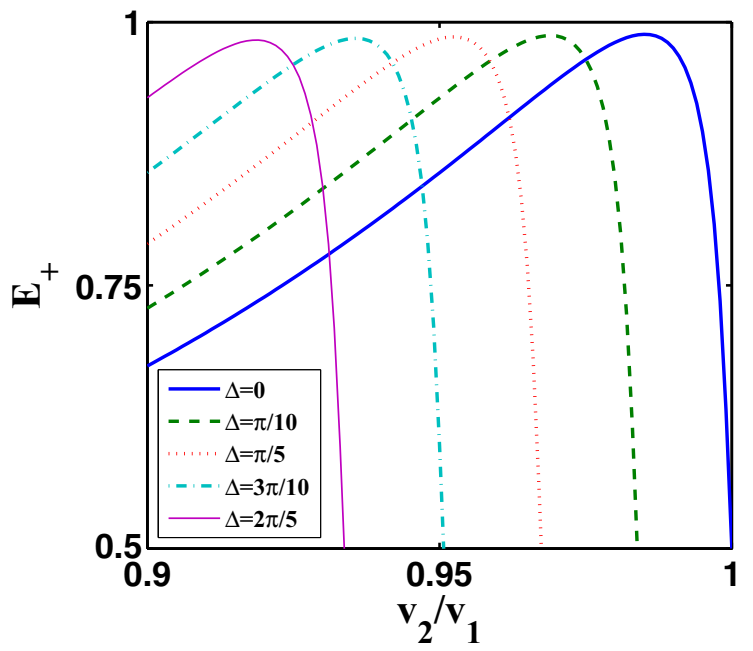

(a)

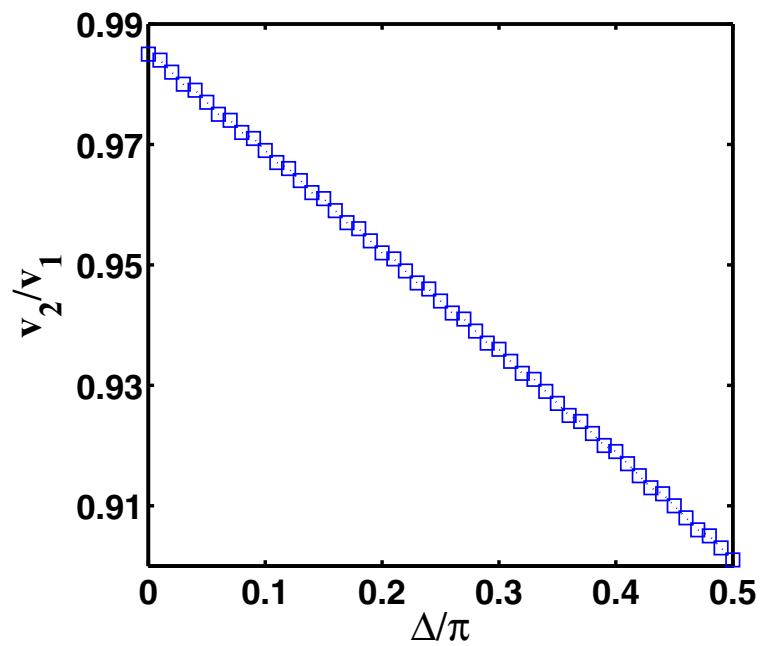

(b)

FIG. 4. (Color online) The top panel shows the variation of the fraction $E_{+}$as a function of velocity for different relative phases between the bright solitons. The bottom panel shows the shift of the optimal point (of maximal asymmetry) as a function of the relative phase $\Delta$. The other parameters are chosen as $q=1, \sigma=0.1, k_{1}=$ $k_{2}=1, v_{1}=1$, and $x_{1} / v_{1}=x_{2} / v_{2}=20$.

effect observed above still persists as in Fig. 3. We consider two bright solitons starting at $\pm x_{0}$, and they collide at the approximate position $x=x_{0}\left(\frac{1-v_{2} / v_{1}}{1+v_{2} / v_{1}}\right)$. This situation is similar to the case when $v_{2} / v_{1} \rightarrow 1$, although the maximal asymmetry may occur, e.g., as $v_{2} / v_{1} \rightarrow 0.9$. With our parameters $\left(x_{0}=\right.$ 20 ), the collision position is $x \approx 1.05$ when $v_{2} / v_{1}=0.9$, and parts of the colliding bright solitons are still within the scale of the Gaussian barrier (for $k_{1}=k_{2}=1$ ). Our simulation shows that the difference on $E_{+}$is small as $v_{2} / v_{1} \rightarrow 0.9$, and smaller especially for the slow bright solitons.

A particularly interesting variation of the theme is that asymmetries in bright soliton amplitudes/width may also be used to produce a complete asymmetry (in either direction) of the collisional outcome. This is illustrated by varying $k_{1}$ while keeping $k_{2}$ fixed and $\left|\frac{k_{2}-k_{1}}{k_{1}}\right| \leqslant 0.1$ in our simulations shown in Fig. 5. In panel (a), we give a group of results for the bright

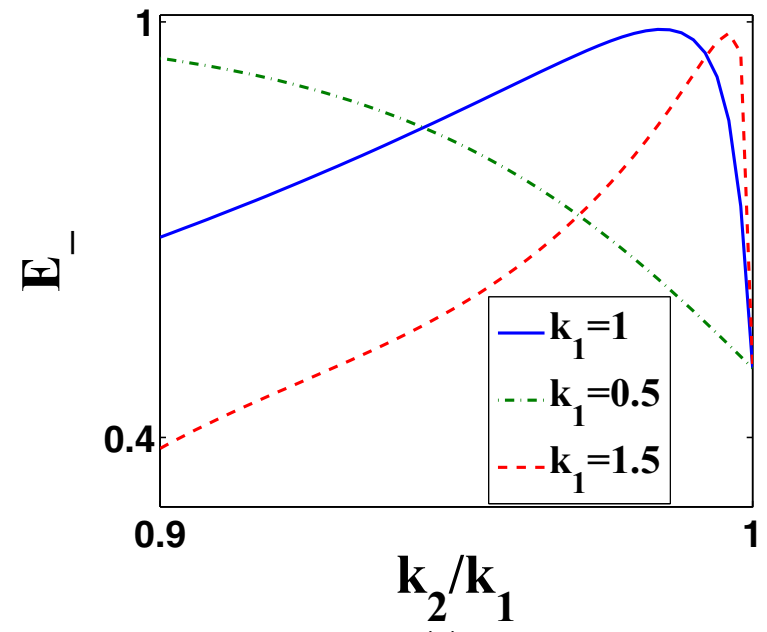

(a)

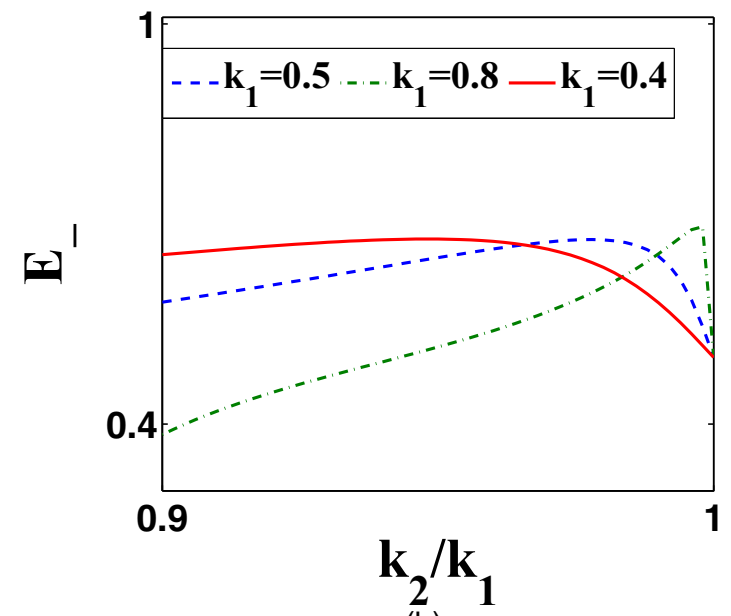

(b)

FIG. 5. (Color online) Plots of $E_{-}$with $k_{2} / k_{1}$ varying from 0.9 to 1.0 for two groups of three values of $k_{1}$. The parameters are chosen as $q=1, \sigma=0.1, \Delta=0$, and $x_{1} / v_{1}=x_{2} / v_{2}=20$. (a) $v_{1}=v_{2}=1$; (b) $v_{1}=v_{2}=0.2$.

soliton collision with $v_{1}=v_{2}=1$. The variation of amplitude (or inverse width) distribution with $k_{2} / k_{1}$ is similar to that with $v_{2} / v_{1}$ after collision. However, for bright solitons with smaller amplitude and larger width (our simulations are based on bright solitons with amplitude comparable to the barrier height, and width much larger than the barrier width), the curve $E_{-}$is more flat and the effect is considerably less pronounced, especially so for slower bright solitons; cf. Fig. 5(b).

Another key element in the analysis of this HOM-type phenomenology is the role of the "beam splitter," i.e., how the observations are modified by varying the parameters of the barrier. To analyze that, we fix $v_{1}=1$ and give two simulation results in Fig. 6 with variation of the barrier strength and width. Equation (4) suggests that when $\sigma \ll 0.8$ (for $q=1$ ), the bright soliton steps into the tunneling regime. Figure 6(a) shows that the curve $E_{+}$is gradually approaching a constant value of 0.5 as $\sigma \geqslant 1$, which can be seen as being outside the tunneling regime. On the other hand, as $\sigma$ approaches 0.1 , the mass asymmetry is maximized, reaching $\approx 98 \%$ in one detector. However, if we further decrease the width, the 

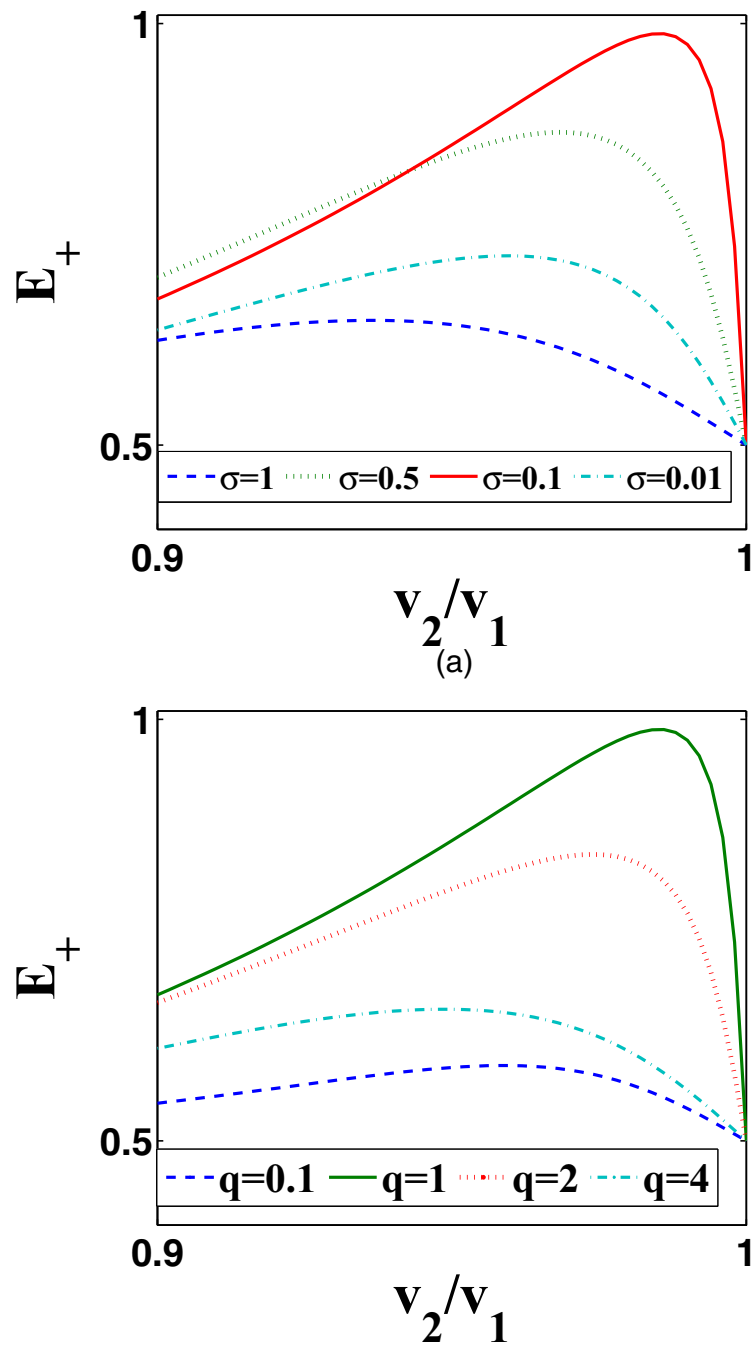

(b)

FIG. 6. (Color online) (a) Plots of $E_{+}$with $v_{2} / v_{1}$ varying from 0.9 to 1.0 for four values of $\sigma(q=1)$. (b) Plots of $E_{+}$with $v_{2} / v_{1}$ varying from 0.9 to 1.0 for four values of $q(\sigma=0.1)$. The parameters are chosen as $k_{1}=k_{2}=1, v_{1}=1, \Delta=0$, and $x_{1} / v_{1}=x_{2} / v_{2}=20$.

maximal asymmetry decreases, indicating a nonmonotonic dependence. The situation is similar (and again nonmonotonic) with variation of $q$. When $q \gg 0.13(\sigma=0.1)$, the bright soliton is considered to be in the tunneling regime. Figure 6(b) shows that, when $q<0.1$, the curve is gradually approaching the constant value of 0.5 again. The maximum asymmetry of our HOM-like detection can be observed as $q$ approaches 1 (i.e., to $v_{1}$ ). Finally, further increase of $q$ beyond the above maximum yield value of $v_{1}$ leads anew to a less pronounced phenomenology and to a flattening of the relevant curve.

To examine the role of potential fluctuations (and asymmetries) of the beam splitter itself (rather than of the bright solitons), we have also examined the possibility of adding random noise on top of the Gaussian barrier in Eq. (1). This was implemented as $V(x)=\frac{q}{\sigma \sqrt{2 \pi}}[1+\eta \varepsilon(x)] e^{-\frac{x^{2}}{2 \sigma^{2}}}$, where $\eta$ is the noise strength, and $\varepsilon(x)$ is a random function with uniformly distributed random values in $[-1,1]$. For the weak noise $(\eta=0.1)$, we have performed simulations in the case of faster $\left(v_{1}=1,12\right.$ realizations) and slower $\left(v_{1}=0.2\right.$, six realizations) solitary waves, respectively. From our observations, we conclude that our findings are only weakly affected by this type of slight variations or asymmetries of the barrier. It is instead chiefly the weak asymmetry of the solitary waves, in the appropriate parametric regime as per the discussion above, that is responsible for the observed phenomenology.

Lastly, in order to offer a glimpse of theoretical insight towards the numerical observations presented herein, we propose the following heuristic argument, which we have tested to be valid for large speeds of the incoming solitary waves. In this case, our observations here appear to capture the slight asymmetry between the incoming solitary waves, building a relative phase difference $\Delta$ between them. The latter, in turn, and in agreement with the arguments of [18], would provide a maximal asymmetry in the collisional output if it assumes the value of $\Delta=\pi / 2$. However, if we assume that our solitary waves are fast enough, then their accumulated phase difference (over the time $t=x_{1} / v_{1}$ needed to reach the barrier collision point) is $\Delta=\left(v_{1}^{2}-v_{2}^{2}\right) t / 2$, as stems from the expression of integrable bright solitons of the GP equation. Setting these two expressions for $\Delta$ equal, we retrieve an estimation of the optimal asymmetry in the form of $\left(v_{2} / v_{1}\right)^{2}=$ $1-\pi /\left(x_{1} v_{1}\right)$. While this is a relatively simplistic calculation, it qualitatively agrees with our numerical computations. In particular, it has prompted us to examine the dependence of the point of optimally asymmetric output on the starting bright soliton location(s), i.e., on $x_{1}$. An example of this is shown in Fig. 7. Indeed, the latter clearly displays the existence of a monotonically decreasing asymmetry trend in the location of the optimum as $x_{1}$ is increased. Moreover, for the large speeds used in this example, the second panel of the figure illustrates that the agreement for the prediction of the relevant optimal asymmetry is not merely qualitative but also quantitative.

\section{CONCLUSIONS AND FUTURE CHALLENGES}

In the present work, we have proposed a mean-field experiment with bright solitons interacting with each other, at a Gaussian barrier, in a way analogous to the Hong-Ou-Mandel experiment with photons (or more generally, bosons). In this analogy, the role of the photons is played by the bright solitons and that of the beam splitter by a Gaussian barrier. Our findings are rather unexpected in many ways. In the limit of perfect symmetry, the output result is, as expected classically (and based on symmetry), an even mass split between the two output ports. On the other hand, in a way somewhat reminiscent of the quantum-mechanical analog, for weak deviations from "indistinguishability," the mean-field treatment of the bright solitons leads to a strongly asymmetric result, one of a nearly perfect $|2,0\rangle$ or $|0,2\rangle$ state. We have quantified this effect and have illustrated its occurrence both for (weak) asymmetries of incoming bright soliton velocities or even for those of bright soliton amplitudes (or inverse widths). This phenomenology has been quantified over variations of parameters of the barrier (such as its strength and inverse width) and relevant optima have been revealed (e.g., when the strength of the barrier is nearly comparable to the incoming bright soliton velocities, i.e., in the nearly 50:50 beam-splitter regime). 


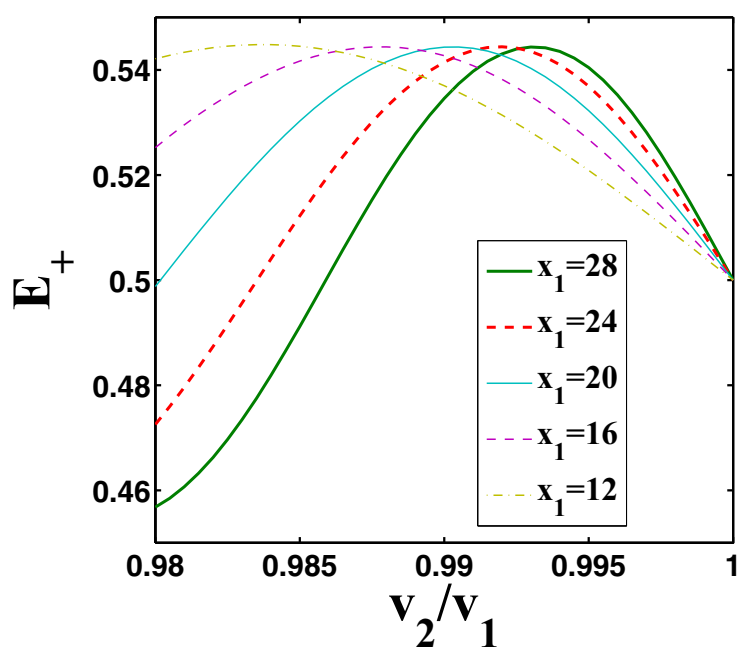

(a)

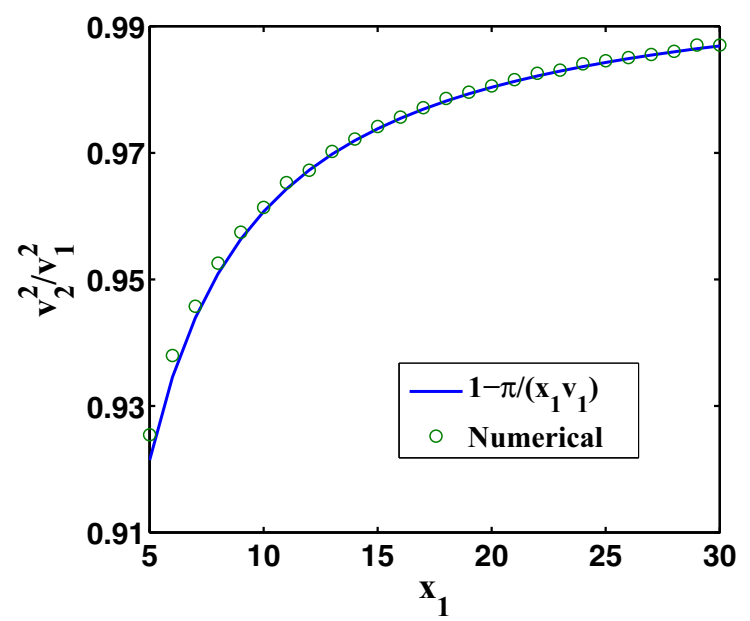

(b)

FIG. 7. (Color online) (a) Plots of $E_{+}$as a function of $v_{2} / v_{1}$ for five distinct values of $x_{1}$. The remaining parameters are $q=1, \sigma=$ $0.1, k_{1}=k_{2}=1$, and $v_{1}=8$. (b) The point of optimal asymmetry quantified by $\left(v_{2} / v_{1}\right)^{2}$ is shown as a function of $x_{1}$ together with the corresponding theoretical prediction of our heuristic argument; see the discussion of the last paragraph of Sec. III. Notice that these panels are constructed for a large value of speed where, as is illustrated, this argument is quantitatively valid.

These results pave the way for a considerable number of additional investigations in this field. On the one hand, from a more mathematical perspective, it becomes especially relevant to consider the appropriate extension of the work of [33] and a potentially deeper and more quantitative understanding of the role of asymmetries in multisoliton collisions (with a barrier). On the other hand, from a physical perspective, it would be especially interesting to explore whether different wave entities feature similar behavior upon their interactions with barriers. For instance, in the context of repulsive condensates, it would be interesting to examine whether weakly asymmetric dark solitons [34] or perhaps even asymmetric vortices [35] may yield similar features in their pairwise interactions with barriers. The fact that such interactions in both one- and even in multicomponent settings have recently started to be considered [36] suggests the relevance of such studies. On the other hand, multicomponent variants of the problem would be worthwhile to explore, even in the self-attractive case, where in addition to the potential asymmetry in output ports, further asymmetries between components can be envisioned.

Another, potentially rather challenging direction may involve the recently analyzed analogy between the Lieb-Liniger exactly solvable model 1D solutions and their mean-field bright solitonic counterparts in the larger atom number limit [37] to explore the question discussed herein for structures involving different atom numbers. As this control parameter decreases, we can gradually progress from the mean-field limit of the present work to the quantum-mechanical realm of the Lieb-Liniger model and examine how the latter may modify the presently reported phenomenology. These topics are currently under consideration and will be presented in future work.

\section{ACKNOWLEDGMENTS}

Z.-Y.S. acknowledges partial support from Technion in the form of a fellowship from the Israel Council for Higher Education. Z.-Y.S. also thanks the Department of Mathematics and Statistics at UMass Amherst for the hospitality during his visit there. P.G.K. acknowledges support from the National Science Foundation under Grants No. CMMI-1000337 and No. DMS-1312856, from the ERC and FP7-People under Grant No. IRSES-605096, from the Binational (US-Israel) Science Foundation through Grant No. 2010239, and from the US-AFOSR under Grant No. FA9550-12-10332. P.G.K. is also grateful to J. Stockhofe for numerous informative discussions on the HOM experiment and related themes, as well as to J. Cuevas for discussions at the early stages of this project. P.K. acknowledges support by the EPSRC (Grant No. EP/I017828/1) and the European Commission (Grant No. FP7-ICT-601180). P.G.K. and P.K. also acknowledge the hospitality of the Synthetic Quantum Systems group and of Markus Oberthaler at the Kirchhoff Institute for Physics (KIP) at the University of Heidelberg, as well as that of the Center for Optical Quantum Technologies (ZOQ) and of Peter Schmelcher at the University of Hamburg.
[1] C. K. Hong, Z. Y. Ou, and L. Mandel, Phys. Rev. Lett. 59, 2044 (1987).

[2] C. Santori, D. Fattal, J. Vučković, G. S. Solomon, and Y. Yamamoto, Nature (London) 419, 594 (2002).

[3] J. Beugnon, M. P. A. Jones, J. Dingjan, B. Darquié, G. Messin, A. Browaeys, and P. Grangier, Nature (London) 440, 779 (2006).
[4] E. Knill, R. Laflamme, and G. J. Milburn, Nature (London) 409, 46 (2001).

[5] Y. L. Lim and A. Beige, New J. Phys. 7, 155 (2005).

[6] P. Longo, J. H. Cole, and K. Busch, Opt. Express 20, 12326 (2012)

[7] F. Laloë and W. J. Mullin, Found. Phys. 42, 53 (2012). 
[8] R. J. Lewis-Swan and K. V. Kheruntsyan, Nat. Commun. 5, 3752 (2014).

[9] K. V. Kheruntsyan, J.-C. Jaskula, P. Deuar, M. Bonneau, G. B. Partridge, J. Ruaudel, R. Lopes, D. Boiron, and C. I. Westbrook, Phys. Rev. Lett. 108, 260401 (2012).

[10] G. Di Martino, Y. Sonnefraud, M. S. Tame, S. Kéna-Cohen, F. Dieleman, S. K. Özdemir, M. S. Kim, and S. A. Maier, Phys. Rev. Applied 1, 034004 (2014).

[11] P. G. Kevrekidis, D. J. Frantzeskakis, and R. CarreteroGonzález, Emergent Nonlinear Phenomena in Bose-Einstein Condensates: Theory and Experiment, Springer Series on Atomic, Optical, and Plasma Physics Vol. 45 (Springer, Berlin, 2008).

[12] L. Khaykovich, F. Schreck, G. Ferrari, T. Bourdel, J. Cubizolles, L. D. Carr, Y. Castin, and C. Salomon, Science 296, 1290 (2002).

[13] K. E. Strecker, G. B. Partridge, A. G. Truscott, and R. G. Hulet, Nature (London) 417, 150 (2002).

[14] K. E. Strecker, G. B. Partridge, A. G. Truscott, and R. G. Hulet, New J. Phys. 5, 73 (2003).

[15] S. L. Cornish, S. T. Thompson, and C. E. Wieman, Phys. Rev. Lett. 96, 170401 (2006).

[16] F. Kh. Abdullaev, A. Gammal, A. M. Kamchatnov, and L. Tomio, Int. J. Mod. Phys. B 19, 3415 (2005).

[17] T. P. Billam, A. L. Marchant, S. L. Cornish, S. A. Gardiner, and N. G. Parker, Bright Solitary Matter Waves: Formation, Stability and Interactions in Spontaneous Symmetry Breaking, Self-Trapping, and Josephson Oscillations, edited by B. A. Malomed (Springer-Verlag, New York, 2013).

[18] J. L. Helm, T. P. Billam, and S. A. Gardiner, Phys. Rev. A 85, 053621 (2012).

[19] J. Cuevas, P. G. Kevrekidis, B. A. Malomed, P. Dyke, and R. G. Hulet, New J. Phys. 15, 063006 (2013).

[20] A. D. Martin and J. Ruostekoski, New. J. Phys. 14, 043040 (2012).

[21] B. Gertjerenken, T. P. Billam, L. Khaykovich, and C. Weiss, Phys. Rev. A 86, 033608 (2012).
[22] A. L. Marchant, T. P. Billam, T. P. Wiles, M. M. H. Yu, S. A. Gardiner, and S. L. Cornish, Nat. Commun. 4, 1865 (2013).

[23] A very recent experimental example of interactions in the absence of a barrier is given also in J. H. V. Nguyen, P. Dyke, D. Luo, B. A. Malomed, and R. G. Hulet, Nat. Phys. 10, 918 (2014).

[24] R. Balakrishnan and I. I. Satija, Pramana J. Phys. 77, 929 (2011).

[25] U. Al Khawaja, H. T. C. Stoof, R. G. Hulet, K. E. Strecker, and G. B. Partridge, Phys. Rev. Lett. 89, 200404 (2002).

[26] N. G. Parker, A. M. Martin, S. L. Cornish, and C. S. Adams, J. Phys. B 41, 045303 (2008).

[27] V. Achilleos, D. J. Frantzeskakis, P. G. Kevrekidis, and D. E. Pelinovsky, Phys. Rev. Lett. 110, 264101 (2013).

[28] Y. Xu, Y. Zhang, and B. Wu, Phys. Rev. A 87, 013614 (2013).

[29] O. A. Egorov, D. V. Skryabin, A. V. Yulin, and F. Lederer, Phys. Rev. Lett. 102, 153904 (2009).

[30] M. Sich, F. Fras, J. K. Chana, M. S. Skolnick, D. N. Krizhanovskii, A. V. Gorbach, R. Hartley, D. V. Skryabin, S. S. Gavrilov, E. A. Cerda-Méndez, K. Biermann, R. Hey, and P. V. Santos, Phys. Rev. Lett. 112, 046403 (2014).

[31] P. Medley, M. A. Minar, N. C. Cizek, D. Berryrieser, and M. A. Kasevich, Phys. Rev. Lett. 112, 060401 (2014).

[32] G. D. McDonald, C. C. N. Kuhn, K. S. Hardman, S. Bennetts, P. J. Everitt, P. A. Altin, J. E. Debs, J. D. Close, and N. P. Robins, Phys. Rev. Lett. 113, 013002 (2014).

[33] J. Holmer, J. Marzuola, and M. Zworski, Commun. Math. Phys. 274, 187 (2007); J. Nonlinear Sci. 17, 349 (2007).

[34] D. J. Frantzeskakis, J. Phys. A: Math. Theor. 43, 213001 (2010).

[35] A. L. Fetter and A. A. Svidzinsky, J. Phys.: Condens. Matter 13, R135 (2001); A. L. Fetter, Rev. Mod. Phys. 81, 647 (2009).

[36] A. Álvarez, J. Cuevas, F. R. Romero, C. Hamner, J. J. Chang, P. Engels, P. G. Kevrekidis, and D. J. Frantzeskakis, J. Phys. B 46, 065302 (2013).

[37] B. Gertjerenken, Phys. Rev. A 88, 053623 (2013); see, in particular, Sec. II. 